\title{
Once-Daily Dosing of Gentamicin
}

Gentamicin remains one of the most effective and commonly used antimicrobials in the armamentarium of clinicians treating pelvic infections. It continues to reliably cover the aerobic gram-negative rods seen within polymicrobial infections. Available in its generic form, it becomes the low-cost drug of choice in these settings. So why mess with success?

As Wiesenfield et al ${ }^{1}$ point out in this issue of the Journal, you can and should teach an old dog new tricks. The bactericidal activity of gentamicin is characterized by concentration-dependent killing. The higher the drug concentration, the greater the rate and extent of bactericidal activity. More over, prolonged postantibiotic effects (PAE) are observed in gram-negative bacilli following exposure to antimicrobials that are inhibitors of protein synthesis, such as gentamicin. PAE refers to the persistent suppression of bacterial growth following exposure to an antimicrobial. This PAE is further prolonged, almost doubled in fact, by the presence of leukocytes.

In clinical trials correlating pharmacokinetic/pharmacodynamic parameters of aminoglycosides with therapeutic efficacy, a clinical response of $>90 \%$ required the peak level to exceed the minimum inhibition concentration (MIC) by eightfold to 10-fold. Once-daily dosing with aminoglycosides is designed to enhance peak serum concentrations. Peak concentrations that are eight to 10 times higher than the MIC can reduce the rate of emergence of aminoglycoside-resistant mutants during therapy. In addition, initial exposure of bacteria to aminoglycosides downregulates subsequent uptake of the drug. During this downregulation, decreased killing of bacteria and higher MICs are noted. Once-daily dosing allows time for this effect to dissipate between doses. ${ }^{2}$

Finally, once-daily dosing has the potential to reduce the incidence of nephrotoxicity and ototoxicity associated with the use of aminoglycosides. Uptake of these drugs into the renal tubular cells and endolymph of the ear is more efficient with low sustained concentrations than with high intermittent levels. It also appears that toxicity is delayed in those patients dosed once a day. Given the typical short-term, commando-type use of gentamicin in the treatment of pelvic infections, one could use the agent, expect a response, and discontinue therapy promptly with less concern for toxicity. Measuring aminoglycoside levels is not necessary in most of the patients we treat, allowing for additional cost savings.

Gentamicin use in the combination therapy for pelvic infections has a solid track record of efficacy. Once-daily gentamicin dosing would appear to have an edge in an environment of cost consciousness and safety.

\section{David E. Soper, MD \\ Medical University of South Carolina \\ Charleston, $S C$}

\section{REFERENCES}

1. Wiesenfield HC, Heine RP. The use of once-daily dosing of gentamicin in obstetrics and gynecology. Infect Dis Obstet Gynecol 6:155-159, 1998.

2. Craig WA. Pharmacokinetic/pharmacodynamic parameters: Rationale for antibacterial dosing of mice and men. Clin Infect Dis 26:1-12, 1998. 


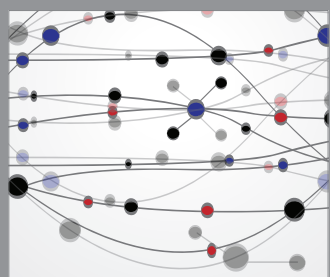

The Scientific World Journal
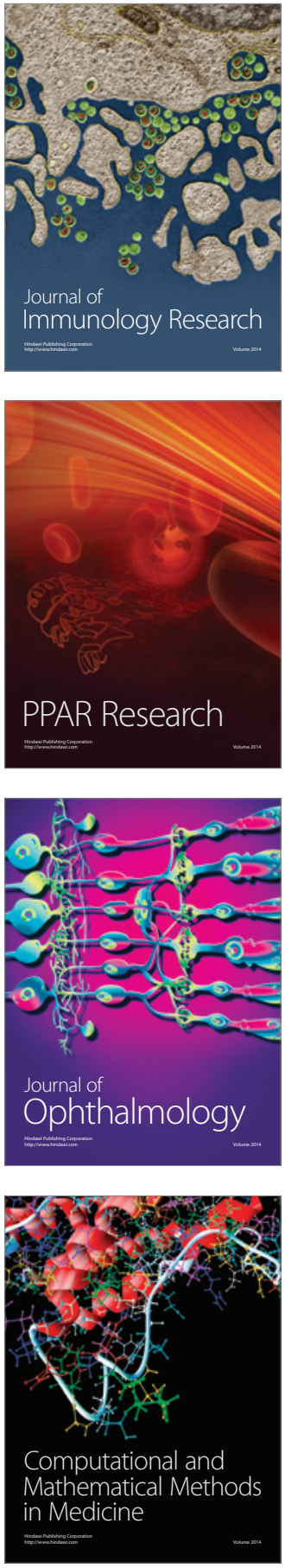

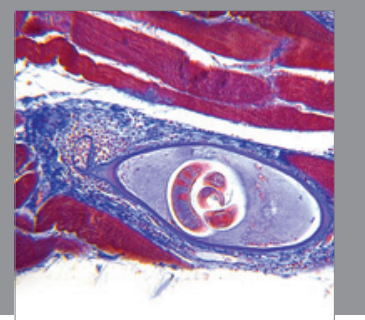

Gastroenterology

Research and Practice
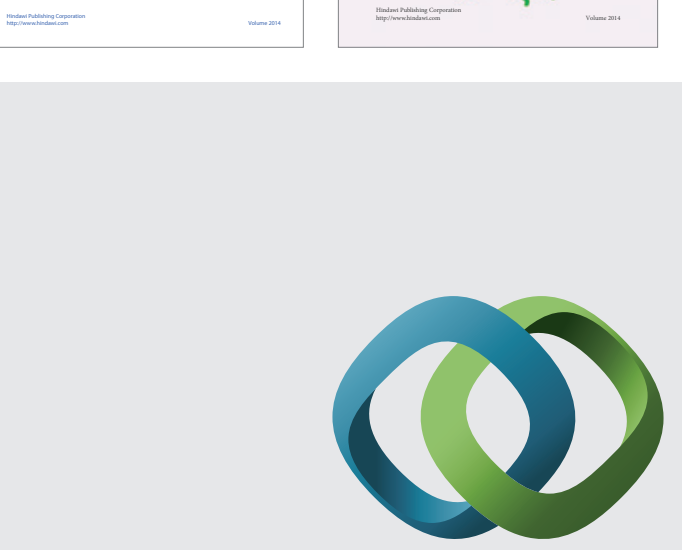

\section{Hindawi}

Submit your manuscripts at

http://www.hindawi.com
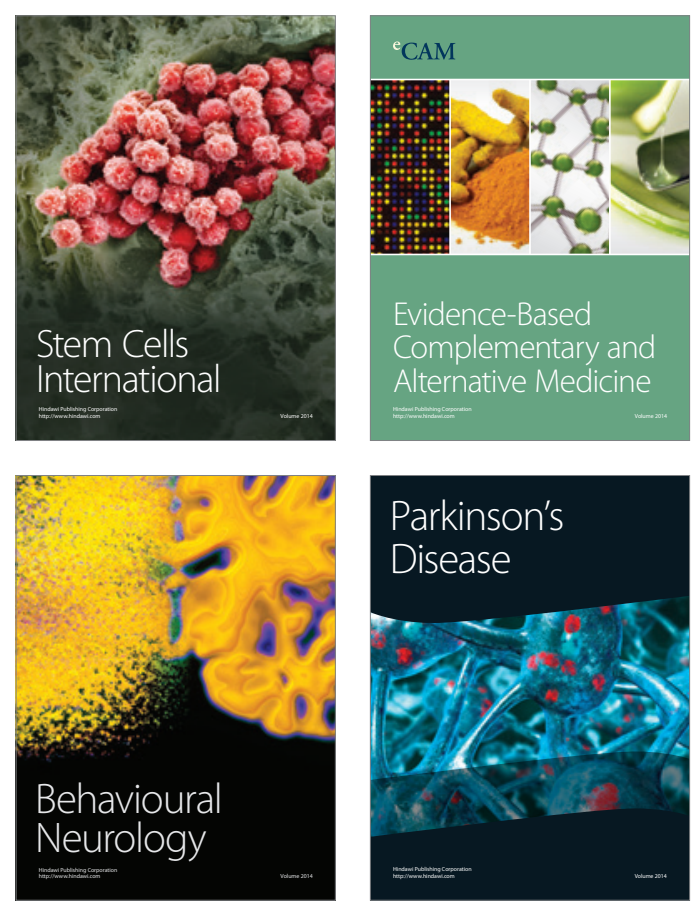

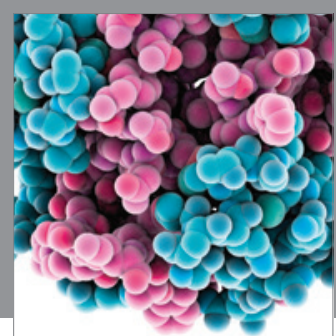

Journal of
Diabetes Research

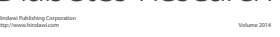

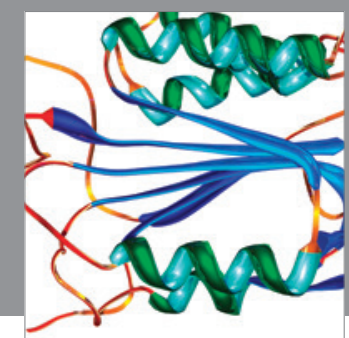

Disease Markers
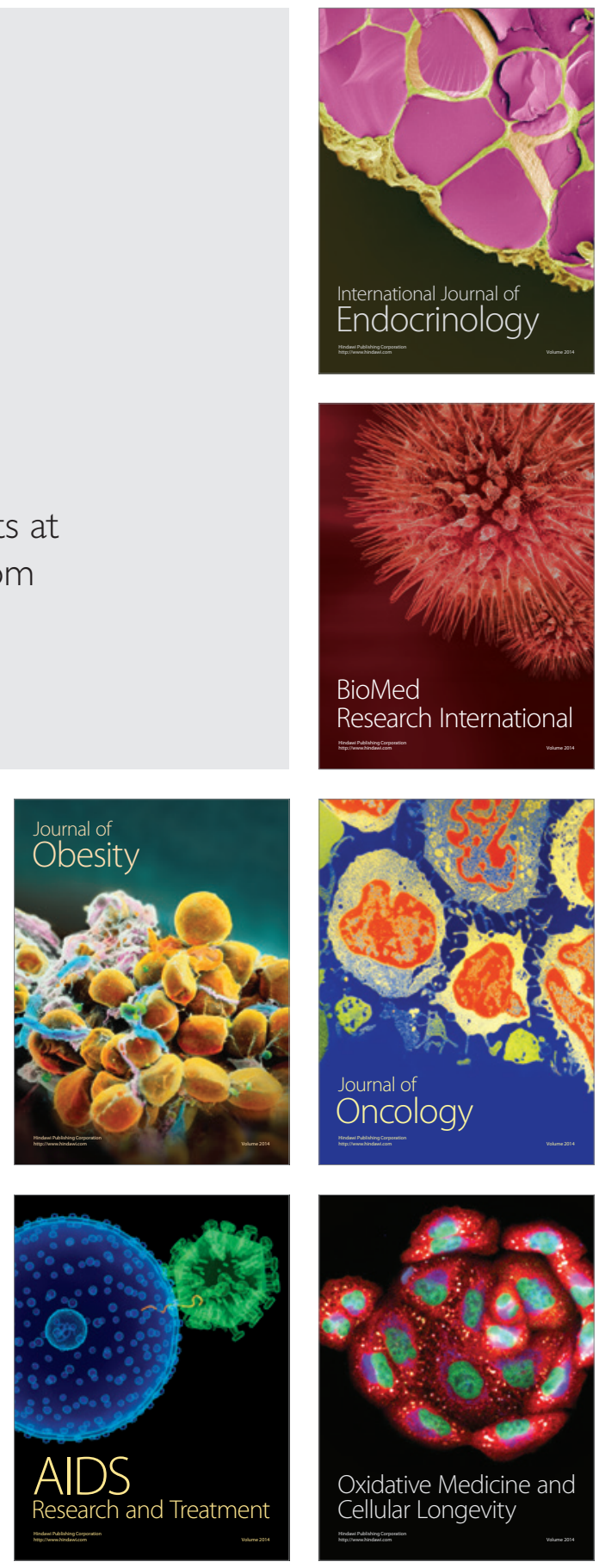